Nelsilene Mota Carvalho Tavares ${ }^{1}$

SABRINA Girotto Ferreira ${ }^{1}$

JoÃo Renato Bennin ${ }^{1}$

Emílo Francisco Marussi ${ }^{1}$

RICARDO BARINI ${ }^{1}$

Cleisson Fábio Andriol Peralta ${ }^{1}$

Artigo Original

Palavras-chave

Fluxometria por laser-doppler

Valores de referência

Ultrassonografia doppler/métodos

Estudos longitudinais

Keywords

Laser-doppler flowmetry Reference values

Ultrasonography, doppler/methods Longitudinal studies

Correspondência

Cleisson Fábio Andrioli Peralta Departamento de Ginecologia e Obstetrícia do Centro de Atenç̣̃o Integral à Saúde da Mulher da Universidade Estadual de Campinos Rua Alexander Fleming, 101 - Cidade Universitária Zeferino Vaz

CEP: $13083-970$

Campinas (SP), Brasil

Recebido

20/06/12

Aceito com modificacoōes

$06 / 11 / 12$

\section{Intervalos de referência longitudinais de parâmetros doplervelocimétricos materno-fetais}

\author{
Longitudinal reference intervals of maternal-fetal Doppler parameters
}

\section{Resumo}

OBJETIVO: Criar intervalos de referência longitudinais para os valores de índices de pulsatilidade (IP) dos fluxos nas artérias umbilicais (AU), cerebral média (ACM) e uterinas (AUt) e IP venoso do fluxo no ducto venoso (DV) com uma amostra da população brasileira. MÉTODOS: Estudo observacional longitudinal realizado de fevereiro de 2010 a maio de 2012. Gestantes de baixo risco foram submetidas a exames ultrassonográficos quinzenais da $18^{\circ}$ a $40^{\circ}$ semana para obtenção dos IP das $A U$, AUt, ACM e IP venoso do DV. Modelos lineares mistos foram usados para elaboração de intervalos de referência longitudinais (percentis 5, 50 e 95) dos IP dos vasos mencionados. Os IP das porções placentária e abdominal do cordão umbilical foram comparados por meio do teste $t$ de amostras independentes. Valores de p bilaterais menores do que 0,05 foram considerados significativos. RESULTADOS: Cento e sessenta e quatro gestantes foram submetidas a 1.242 exames ultrassonográficos. Houve redução significativa nos valores de todos esses parâmetros com o avançar da IG. Entre a $18^{a}$ e a 40 semana de gravidez, as medianas de IP da AU (porções abdominal e placentária do cordão), da ACM, do DV e do IP médio das AUt variaram de 1,19 a 0,74; 1,33 a 0,78; 1,56 a 1,39; 0,58 a 0,41; e 0,98 a 0,66, respectivamente. As equações obtidas para predição das medianas foram: IP-AU=1,5602786 - (0,020623 x IG); Logaritmo do IP-ACM=0,81491 11 - 10,004168 x IG) - [0,002543 x (IG - 28,7756)²]; Logaritmo do IP-DV=-0,2669 1- (0,015414 x IG); IP-AUt=1, $2362403-(0,014392$ $\times$ (G). Houve diferença significativa entre os IP-AU obtidos nas extremidades placentária e abdominal fetal ( $p<0,001)$. CONCLUSÃO: Foram estabelecidos intervalos de referência longitudinais dos parâmetros doplervelocimétricos gestacionais mais importantes em uma amostra da população brasileira. Estes podem ser mais adequados para o acompanhamento das modificações hemodinâmicas materno-fetais em gestações normais ou não, o que ainda requer validação futura.

\section{Abstract}

PURPOSE: To create longitudinal reference intervals for pulsatility index (PI) of the umbilical (UA), middle cerebral (MCA), uterine (UtA) arteries and ductus venosus (DV) in a Brazilian cohort. METHODS: A longitudinal observational study performed from February 2010 to May 2012. Low risk pregnancies were scanned fortnightly from 18 to 40 weeks for the measurements of Pl of the UA, MCA, DV and UtA. Linear mixed models were used for the elaboration of longitudinal reference intervals (5th, 50th and 95th percentiles) of these measurements. Pl obtained for the placental and abdominal portions of the umbilical artery were compared by the t-test for independent samples. Two-sided $p$ values of less than 0.05 were considered statistically significant. RESULTS: A total of 164 patients underwent 1,242 scans. There was significant decrease in PI values of all vessels studied with gestational age (GA). From the $18^{\text {th }}$ to the $40^{\text {th }}$ week of pregnancy, the median PI values of UA (abdominal and placental ends of the cord), MCA, DV and the mean Pl of the UtA ranged from 1.19 to $0.74,1.33$ to $0.78,1.56$ to 1.39, 0.58 to 0.41 , and 0.98 to 0.66 , respectively. The following equations were obtained for the prediction of the medians: PI-UA=1.5602786-(0.020623 x GA); Logarithm of the PI-MCA=0.81491 $11-(0.004168 \times \mathrm{GA})-\left[0.02543 \times(\mathrm{GA}-28.7756)^{2}\right]$; Logarithm of the PI-DV=-0.2669 1(0.015414 × GA); PI-UtA = $1.2362403-(0.014392 \times$ GA). There was a significant difference between the PI-UA obtained at the abdominal and placental ends of the umbilical cord ( $p<0.001$ ). CONCLUSIONS: Longitudinal reference intervals for the main gestational Doppler parameters were obtained in a Brazilian cohort. These intervals could be more adequate for the followup of maternalffetal hemodynamic modifications in normal and abnormal pregnancies, a fact that still requires further validation.

Trabalho realizado no Centro de Atenção Integral à Saúde da Mulher da Universidade Estadual de Campinas - UNICAMP Campinas (SP), Brasil.

'Departamento de Obstetrícia e Ginecologia, Centro de Atenção Integral à Saúde da Mulher - CAISM, Universidade Estadual de Campinas - UNICAMP - Campinas (SP), Brasil. 


\section{Introdução}

A doplervelocimetria tem sido um dos principais pilares na avaliação do bem-estar fetal, principalmente por meio da obtenção dos índices de pulsatilidade (IP) dos fluxos nas artérias uterinas (AUt), umbilicais (AU), cerebrais médias (ACM) e no ducto venoso (DV) ${ }^{1-13}$. Estes permitem a respectiva avaliação das condições hemodinâmicas nos territórios vasculares uteroplacentário (AUt) e feto-placentário (AU, ACM e DV) $)^{1-13}$.

A interpretação de cada um desses parâmetros tem sido feita rotineiramente com base em intervalos de referência transversais, que podem não refletir de forma adequada as adaptações maternas e/ou fetais ao longo da gravidez ${ }^{1-3,8,11-13}$. Intervalos de referência (sinônimo mais apropriado para as chamadas curvas de normalidade) representam variações de medidas obtidas de indivíduos saudáveis. Podem ser elaborados por meio de estudos transversais (cada sujeito da pesquisa é avaliado/medido uma única vez) ou longitudinais (cada sujeito é avaliado em mais de uma ocasião, ou seja, acompanhado ao longo de um determinado período). Os intervalos de referência longitudinais (também chamados de intervalos de referência condicionais) têm a finalidade de permitir a interpretação das modificações de determinados parâmetros ao longo do tempo.

Como as características hemodinâmicas materno-fetais em situações de alto risco apresentam caráter evolutivo, os parâmetros doplervelocimétricos durante estas gestações poderiam ser mais bem interpretados à luz de intervalos de referência longitudinais ${ }^{4-6,9}$. Além disso, pouco se sabe se diferenças populacionais ou étnicas podem interferir nos intervalos de referência dos parâmetros doplervelocimétricos gestacionais ${ }^{8}$. Em nosso país, os intervalos mais utilizados são os de Arduini e Rizzo ${ }^{1}$, obtidos de estudos transversais.

Assim sendo, o objetivo deste estudo foi criar intervalos de referência longitudinais dos principais parâmetros doplervelocimétricos usados na gestação, com uma amostra da população brasileira.

\section{Métodos}

Este foi um estudo observacional descritivo longitudinal realizado no Hospital da Mulher Professor Dr. José Aristodemo Pinotti, Centro de Atenção Integral à Saúde da Mulher (CAISM), de fevereiro de 2010 a maio de 2012. O Comitê de Ética em Pesquisa da Universidade Estadual de Campinas (UNICAMP) aprovou o projeto e todas as pacientes que aceitaram participar do estudo assinaram o termo de consentimento livre e esclarecido.

As gestantes foram selecionadas entre aquelas encaminhadas do pré-natal de baixo risco do CAISM para ultrassonografia obstétrica. Em sua maioria, são mulheres de etnia mista e provenientes de classes sociais menos favorecidas, que se beneficiam de atendimento no sistema público de saúde. Os seguintes critérios foram respeitados para inclusão no estudo: gestação única; idade gestacional (IG) entre a $18^{\mathrm{a}}$ e a $24^{\mathrm{a}}$ semana, definida com base na data da última menstruação (quando conhecida) ou na medida do comprimento céfalo-caudal fetal no primeiro trimestre da gravidez, interpretado em intervalos de referência publicados por Robinson e Fleming ${ }^{14}$; anatomia fetal normal durante os exames ultrassonográficos obstétricos realizados até a inclusão no estudo; ausência de alterações placentárias como placenta prévia, circunvalada, com suspeita de acretismo ou de qualquer tipo de tumor; ausência de alterações no cordão umbilical, como número alterado de vasos, nós, tumores ou inserção velamentosa na placenta; ausência de doenças maternas ou condições associadas a alterações no desenvolvimento fetal, como pré-eclâmpsia, diabetes, uso de drogas lícitas ou não.

Foram utilizados os seguintes critérios para exclusão de pacientes do estudo: qualquer anormalidade estrutural ou cromossômica detectada no feto durante o seguimento ultrassonográfico ou no recém-nascido; qualquer alteração placentária ou de cordão umbilical (mencionada nos critérios de inclusão) detectada durante o seguimento ultrassonográfico ou após o parto; doença materna diagnosticada após a inclusão no estudo; falta em mais de dois exames ultrassonográficos consecutivos; óbito fetal ou neonatal sem causa determinada; desenvolvimento de restrição de crescimento intrauterino associado a oligoâmnio e alterações doplervelocimétricas nas AU; e a impossibilidade de obtenção dos dados do parto e do recém-nascido quando da resolução da gravidez em outra instituição.

Para o cálculo da IG, quando a discrepância entre as estimativas obtidas com o uso da data da última menstruação e da medida do comprimento céfalo-caudal do embrião/feto no primeiro trimestre foi maior do que $8 \%$, o primeiro método foi descartado. Se a diferença obtida fosse menor ou igual a $8 \%$, a data da última menstruação era considerada ${ }^{14}$.

Todos os exames ultrassonográficos foram realizados por três operadores (NMCT, SGF e JRB, com 12, cinco e seis anos de experiência em ultrassonografia obstétrica, respectivamente), por via abdominal, com a gestante em decúbito dorsal elevado (em torno de $30^{\circ}$ ), com equipamento Medison Accuvix V10 equipado com transdutor C2 - 6 (Medison, South Korea). Cada paciente foi agendada para exames quinzenais após a inclusão no estudo e foi informada de que duas faltas consecutivas implicariam em sua retirada do estudo, sem que seu acompanhamento pré-natal na instituição fosse prejudicado. Em cada exame ultrassonográfico, além da avaliação da anatomia fetal, da quantidade de líquido amniótico, das características e da posição da placenta, biometria fetal básica (diâmetro 
biparietal, circunferência craniana, circunferência abdominal, comprimento do fêmur) e doplervelocimetria (AU, AUt, ACM e DV) foram realizados.

A biometria fetal foi realizada de acordo com método universalmente difundido e a estimativa de peso foi obtida com o uso da fórmula proposta por Hadlock et al. ${ }^{15}\left(\log _{10}\right.$ $\mathrm{EFW}=1.3598+0.051 \times \mathrm{AC}+0.1844 \times \mathrm{FL}-0.0037 \times \mathrm{AC} \times \mathrm{BPD})$.

Para avaliação doplervelocimétrica de todos os vasos investigados neste estudo, foram respeitados alguns princípios gerais: inicialmente, a identificação do vaso foi realizada com o uso do Doppler colorido, para que a janela do Doppler pulsátil pudesse ser adequadamente posicionada; o ângulo de insonação (entre a direção do vaso e a do feixe do Doppler pulsátil) foi mantido abaixo de $20^{\circ}$; a amostra de volume (janela do Doppler pulsátil) foi ajustada entre 1,5 e $3 \mathrm{~mm}$, na dependência do tamanho do vaso insonado; o filtro de parede foi mantido o mais baixo possível na dependência do vaso avaliado $(<50 \mathrm{~Hz}$ para o DV e $<100 \mathrm{~Hz}$ para os demais); pelo menos cinco ondas de velocidades de fluxo (ondas do Doppler pulsátil) uniformes foram obtidas para o cálculo do IP (velocidade máxima-velocidade mínima/velocidade média); o índice térmico foi mantido abaixo de 1,5, de acordo com as orientações da Sociedade Internacional de Doppler Perinatal ${ }^{11,16}$.

As avaliações da AU, da ACM e do DV foram realizadas durante completo repouso fetal (incluindo ausência de movimentos respiratórios). A doplervelocimetria da AU foi efetuada em dois locais diferentes do cordão (o mais próximo possível do abdome fetal e o mais próximo possível da placenta). $\mathrm{Na}$ avaliação da ACM e do DV, a amostra volume foi posicionada no terço proximal do vaso (ACM - próximo ao círculo arterial do cérebro; DV - próximo ao seio portal). Para a doplervelocimetria das AUt, a janela do Doppler pulsátil foi posicionada até $2 \mathrm{~cm}$ abaixo ou acima do cruzamento deste vaso com os ilíacos externos. A média dos IP obtidos nas duas AUt foi usada para análise.

As variáveis maternas e perinatais avaliadas neste estudo foram: idade materna por ocasião da inclusão no estudo, paridade, tipo de parto, IG no momento do parto, peso do recém-nascido e Apgar ao quinto minuto. Os dados perinatais foram colhidos dos prontuários médicos das pacientes.

\section{Análise estatística}

O cálculo do número de gestantes necessárias para o desenvolvimento deste estudo foi realizado da seguinte forma: considerando que um estudo transversal requer um número de pacientes $(N t)$, Royston e Wright ${ }^{17}$ estimaram que o número correspondente de indivíduos necessários para um estudo longitudinal ( $\mathrm{N} l$ ) com o mesmo propósito seria de $\mathrm{NI}=\mathrm{Nt} / 2,3$. Sabe-se que um número mínimo de 15 observações em cada semana gestacional é preciso para o cálculo de percentis confiáveis. Assim sendo, levando em consideração um período de seguimento de 23 semanas, os valores obtidos de $\mathrm{Nt}$ e $\mathrm{Nl}$ seriam, respectivamente, $345 \mathrm{e}$ 152 gestantes. Estimando uma taxa de insucesso no acompanhamento dessas gestantes de aproximadamente $20 \%$, o número necessário de participantes no estudo seria $190^{17}$.

As variáveis maternas e perinatais foram descritas com o uso de medianas e limites (variáveis contínuas), frequências absolutas e relativas (variáveis categóricas).

Modelos lineares mistos foram usados para a elaboração de intervalos de referência condicionais (longitudinais) dos valores de IP da AU (porções abdominal e placentária), da ACM, do DV e de IP médio das AUt. Em cada modelo, o valor absoluto do IP ou seu logaritmo foram usados como variáveis dependentes, e a IG e o número do exame (efeito randômico) foram usados como variáveis preditoras ${ }^{18}$. Para cada variável dependente, modelos de até segunda ordem foram testados, sendo o melhor escolhido com base na comprovação da normalidade dos resíduos gerados (testes de Kolmogorov-Smirnov e de Shapiro-Wilks foram utilizados para este fim $)^{18,19}$. Os percentis 5,50 e 95 dos valores condicionais de IP de fluxo de cada vaso foram estabelecidos.

Os valores de IP obtidos nas duas porções do cordão umbilical foram divididos pelo IP esperado (percentil 50) para a IG (calculado por meio do modelo de predição de valores condicionais de IP na porção placentária) para que pudessem ser expressos em múltiplos da mediana e, assim, comparados por meio do teste $t$ de amostras independentes.

A análise estatística foi realizada com os programas SPSS 20.0 (Chicago, Il, USA), Excel para Windows 2007 (Microsoft Corp., Redmond, WA, USA) e JMP 9 (SAS Institute, USA). Diferenças estatisticamente significativas foram consideradas quando os valores de p obtidos foram menores do que 0,05 .

\section{Resultados}

Duzentas e três gestantes foram convidadas a participar do estudo e assinaram o termo de consentimento livre e esclarecido. Dentre elas, 39 (19,2\%) foram descontinuadas por terem faltado a mais de duas avaliações consecutivas $(31 / 39=79,5 \%)$, desenvolveram pré-eclâmpsia $(4 / 39=10,3 \%)$ ou apresentaram restrição de crescimento fetal com alterações doplervelocimétricas na AU e oligoâmnio (4/39=10,3\%).

Entre as demais 164 pacientes que completaram o estudo, 1.242 exames ultrassonográficos foram realizados (mediana de oito exames por paciente: 4-12). As medianas das IG nos momentos de inclusão no estudo e do último exame antes do nascimento foram 20,1 semanas $(18,0-24,4)$ e 37,3 semanas $(29,0-40,7)$, respectivamente. As idades materna e gestacional por ocasião do parto tiveram medianas de 25 anos (13-46) e 39,3 semanas $(35,3-41,9)$, respectivamente. Cento e cinco pacientes $(105 / 164=64 \%)$ tiveram parto por via vaginal e $59(59 / 164=36 \%)$ por operação cesariana. 
Setenta e seis pacientes $(76 / 164=46 \%)$ eram primigestas. As medianas de peso ao nascimento e de Apgar ao quinto minuto foram $3.180 \mathrm{~g}(1.460-4.220)$ e 10 (7-10).

Os intervalos de referência longitudinais de IP da AU (obtidos nas porções abdominal e placentária do cordão), da ACM, do DV e de IP médio das AUt encontram-se

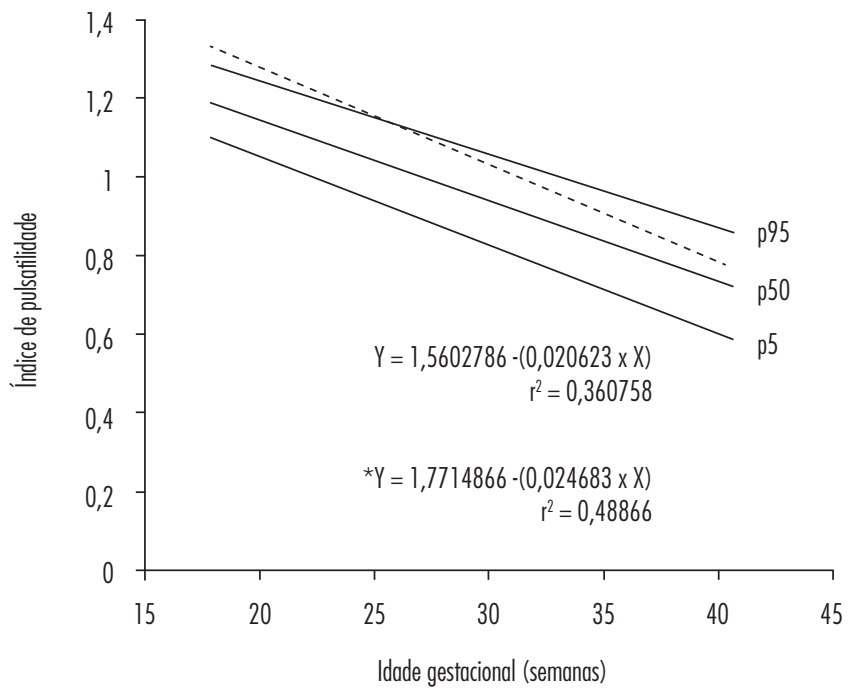

Figura 1. Intervalos de referência longitudinais dos índices de pulsatilidade de fluxo obtidos na artéria umbilical próximo à placenta, de 18 a 40 semanas, com a respectiva fórmula para o cálculo das medianas. A linha pontilhada representa as medianas dos intervalos de referência dos índices de pulsatilidade de fluxo obtidos na artéria umbilical próximo ao abdome fetal, e sua fórmula é antecipada por um * . representados na Tabela 1 , sendo suas respectivas fórmulas expostas nas figuras mencionadas (percentil 50) e na Tabela 2.

Houve redução significativa nos valores de todos esses parâmetros com o avançar da IG. As medianas de IP da AU (porções abdominal e placentária do cordão), da ACM, do DV e do IP médio das AUt entre a $18^{\mathrm{a}}$ e a $40^{\mathrm{a}}$ semana de

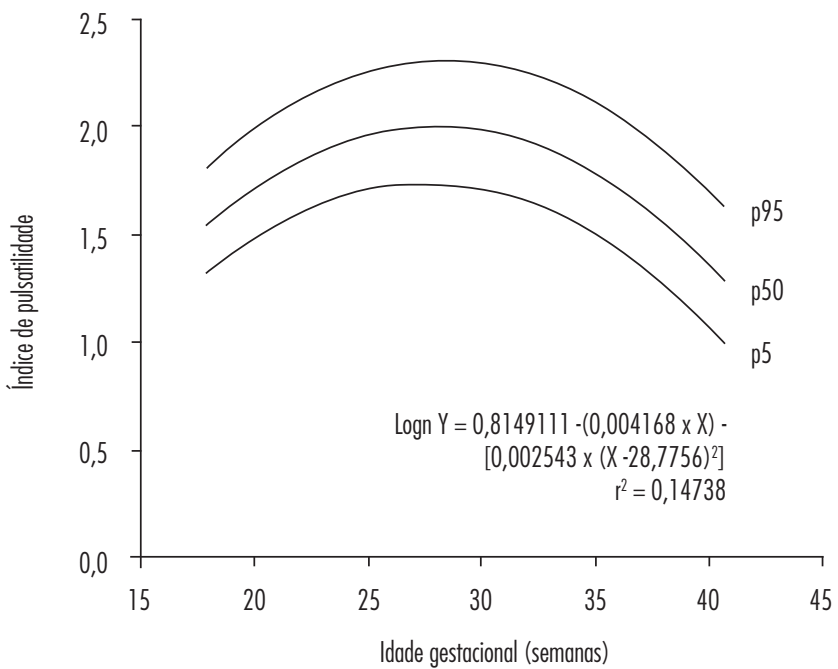

Figura 2. Intervalos de referência longitudinais dos índices de pulsatilidade de fluxo na artéria cerebral média, de 18 a 40 semanas, com a respectiva fórmula para cálculo das medianas.

Tabela 1. Percentis longitudinais 5, 50 e 95 dos índices de pulsatilidade dos fluxos nas artérias umbilical e cerebral média, no ducto venoso e do índice de pulsatilidade médio dos fluxos nas artérias uterinas.

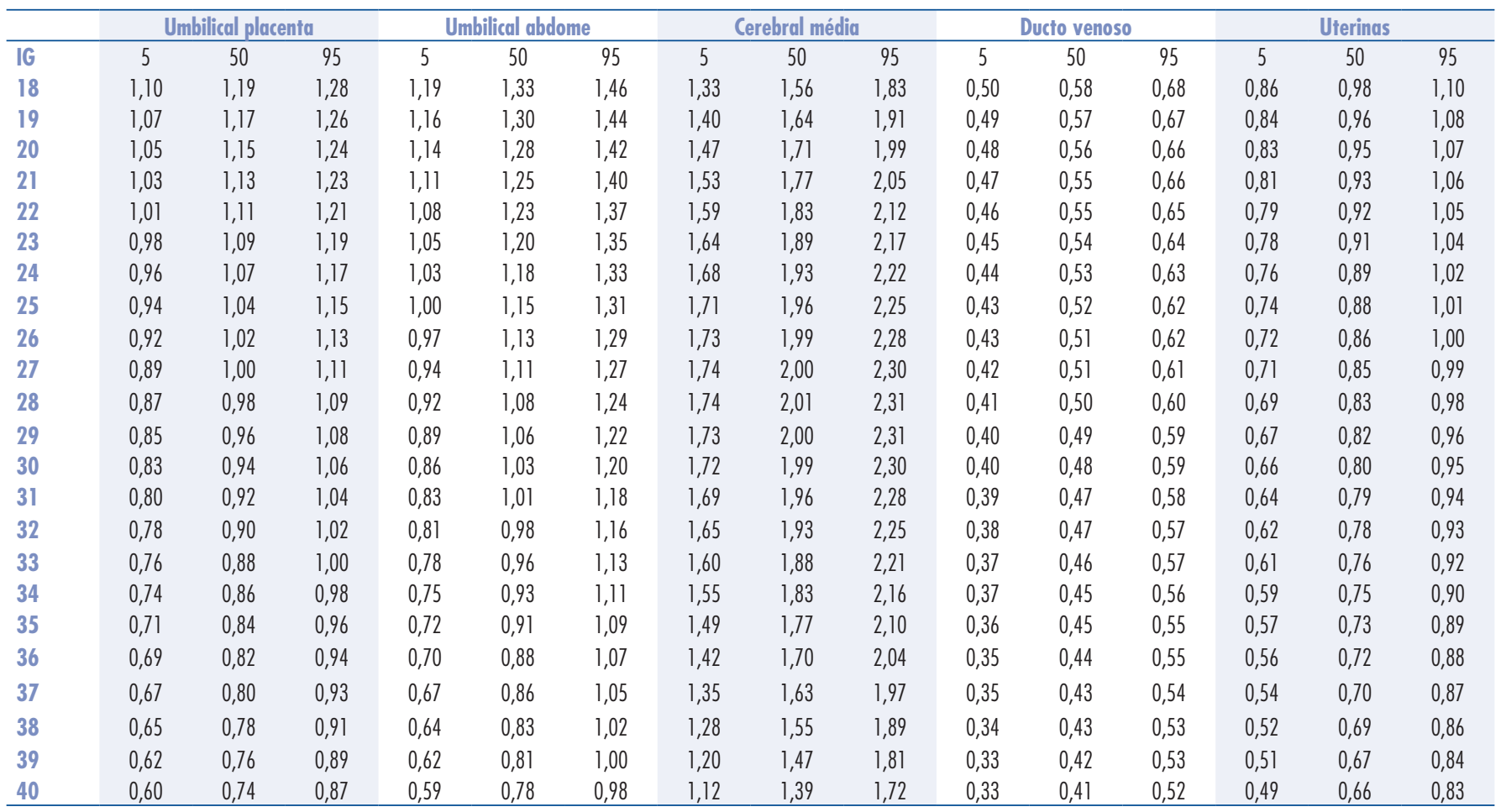




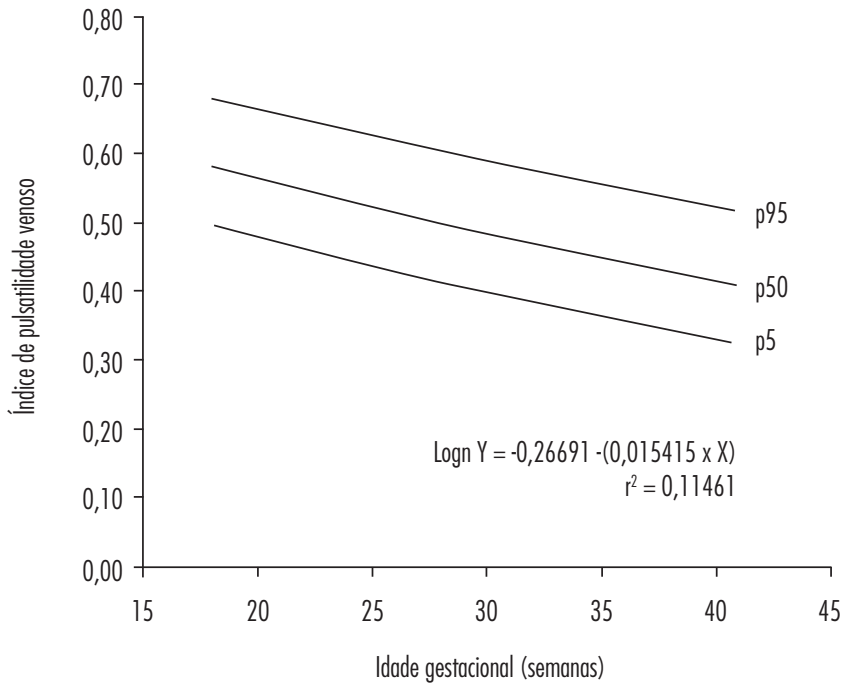

Figura 3. Intervalos de referência longitudinais dos índices de pulsatilidade de fluxo no ducto venoso, de 18 a 40 semanas, com a respectiva fórmula para cálculo das medianas.

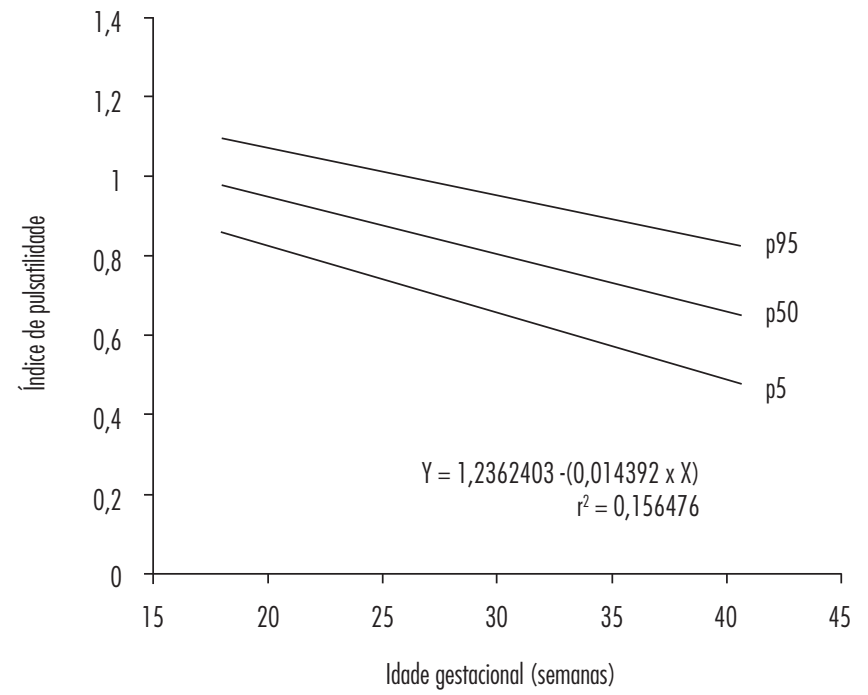

Figura 4. Intervalos de referência longitudinais dos índices de pulsatilidade médios dos fluxos nas artérias uterinas, de 18 a 40 semanas, com a respectiva fórmula para cálculo das medianas.

Tabela 2. Fórmulas obtidas para 0 cálculo dos percentis longitudinais 5 e 95 dos índices de pulsatilidade de fluxo nas artérias umbilical e cerebral média, no ducto venoso e do índice de pulsatilidade médio dos fluxos nas artérias uterinas

\begin{tabular}{|c|c|c|c|}
\hline Vaso & n & Percentil & Equação \\
\hline Artéria umbilical (placenta) & 164 & $\begin{array}{c}5 \\
95\end{array}$ & $\begin{array}{l}\mathbb{P}=1,5021678-(0,022539 \times \mid G) \\
\mathbb{P}=1,6183893-(0,018706 \times \mid G)\end{array}$ \\
\hline Artéria umbilical (abdome) & 164 & $\begin{array}{c}5 \\
95\end{array}$ & $\begin{array}{l}\mathbb{I}=1,6863976-(0,027471 \times \mid \mathrm{G}) \\
\mathbb{I}=1,8565756-(0,021894 \times \mathrm{IG})\end{array}$ \\
\hline Artéria cerebral média & 164 & $\begin{array}{c}5 \\
95\end{array}$ & $\begin{array}{l}\operatorname{Logn} I P=0,7424818-(0,006598 \times I G)-\left[0,00292 \times(I G-28,7756)^{2}\right] \\
\operatorname{Logn} I P=0,8873405-(0,001737 \times \mid G)-\left[0,002165 \times(I G-28,7756)^{2}\right]\end{array}$ \\
\hline Ducto venoso & 119 & $\begin{array}{c}5 \\
95 \\
\end{array}$ & $\begin{array}{l}\operatorname{Logn} I P V=-0,365597-(0,018702 \times \mid G) \\
\operatorname{Logn} I P V=-0,168223-(0,012129 \times \mid G)\end{array}$ \\
\hline Artérias uterinas & 160 & $\begin{array}{c}5 \\
95\end{array}$ & $\begin{array}{l}\mathbb{P}=1,1623672-(0,016838 \times \mathbb{I G}) \\
\mathbb{P}=1,3101135-(0,011946 \times \mathbb{I G})\end{array}$ \\
\hline
\end{tabular}

n: Número de pacientes para os quais a avaliação do vaso foi possível em pelo menos quatro avaliações; IG: Idade gestacional; IP: Índice de pulsatilidade; IPV: Índice de pulsatilidade venoso; Logn: Logaritmo natural;

gravidez variaram de 1,19 a 0,74 , de 1,33 a 0,78 , de 1,56 a 1,39 , de 0,58 a 0,41 e de 0,98 a 0,66 , respectivamente.

Os IP obtidos nas duas porções do cordão umbilical (placentária e abdominal), expressos em múltiplos da mediana (calculada com o modelo de predição de valores condicionais de IP na porção placentária), foram significativamente diferentes $(\mathrm{p}<0,001)$.

\section{Discussão}

Neste estudo, foram estabelecidos intervalos de referência longitudinais para os valores de IP dos fluxos na AU, na ACM, no DV e de IP médio dos fluxos nas AUt, a partir da avaliação sequencial de uma amostra da população brasileira. Foi também demonstrada diferença significativa entre os valores de IP da AU obtidos nas extremidades do cordão.

Um dos principais motivos para a realização deste estudo foi a falta de intervalos de referência longitudinais locais para os parâmetros mencionados. Seria, portanto, adequado que padrões em amostras da nossa população fossem estabelecidos. Outro motivo importante é o fato de que as condições que cursam com alterações doplervelocimétricas na gestação têm caráter evolutivo e demandam avaliações sequenciais das pacientes. Sabe-se que para a interpretação de modificações de quaisquer parâmetros ao longo do tempo, idealmente, intervalos de referência longitudinais devem ser usados. Estes intervalos, diferentes daqueles construídos por meio de estudos transversais, traduzem padrões de modificações ao longo do tempo, e não o estado de um indivíduo em ocasião única. Apesar disso, a interpretação dos parâmetros doplervelocimétricos obstétricos é frequentemente realizada com base em intervalos de referências transversais, que podem não refletir da forma mais precisa as adaptações maternas e/ou fetais ao longo da gravidez ${ }^{1}$.

Apesar de não ter sido objetivo deste trabalho comparar os intervalos de referência aqui gerados com outros disponíveis na literatura, algumas observações e exemplos 
a respeito devem ser mencionados. Comparando-se os intervalos de referência de IP da AU do presente estudo com aqueles obtidos por Acharya et al. ${ }^{4,5}$ (também estudo longitudinal), observa-se semelhança entre os valores das medianas, mas intervalos entre os percentis 5 e 95 maiores no segundo estudo. Esta observação foi comum aos valores de IP da AU obtidos tanto na porção próxima à placenta como na porção próxima ao abdome fetal. De forma semelhante, ao compararmos os intervalos de referência de IP do DV obtidos no presente estudo com os intervalos longitudinais construídos por Kessler et al. ${ }^{6}$, foi observada semelhança entre as medianas, mas discrepância na amplitude entre os percentis 5 e 95, maiores no último trabalho. Essas discrepâncias podem ser parcialmente justificadas pelo número de aferições realizadas em cada estudo. Nos trabalhos de Acharya et al. ${ }^{4}$ e de Kessler et al. ${ }^{6}$, aproximadamente 500 medidas em cada vaso foram realizadas. Em nosso trabalho,
1.242 exames foram efetuados, o que, matematicamente, contribui para o estreitamento entre os percentis. Além disso, não é possível saber se diferenças populacionais podem ter contribuído para estes achados.

Quando nossos dados (IP da AU e da ACM) são confrontados com intervalos de referência transversais classicamente utilizados na prática obstétrica, como os de Arduini e Rizzo ${ }^{1}$, as diferenças em relação aos percentis 5 e 95 ficam ainda maiores (intervalos mais amplos nos estudos transversais).

Um aspecto positivo do presente estudo, portanto, é o número de avaliações realizado em cada paciente, o que não foi observado em outros trabalhos com a mesma finalidade na literatura. Questão que ainda tem de ser respondida é se estes diferentes intervalos de referência têm desempenhos e impactos distintos na detecção e no seguimento dos casos com comprometimento hemodinâmico materno e/ou fetal.

\section{Referências}

1. Arduini D, Rizzo G. Normal values of Pulsatility Index from fetal vessels: a cross-sectional study on 1556 healthy fetuses. J Perinat Med. 1990;18(3): 165-72.

2. Alfirevic Z, Neilson JP. Doppler ultrasonography in high-risk pregnancies: systematic review with meta-analysis. Am J Obstet Gynecol. 1995;172(5):1379-87.

3. Papageorghiou AT, Yu CK, Cicero S, Bower S, Nicolaides KH. Second-trimester uterine artery Doppler screening in unselected populations: a review. J Matern Fetal Neonatal Med. 2002;12(2):7888.

4. Acharya G, Wilsgaard T, Berntsen GK, Maltau JM, Kiserud T. References ranges for serial measurements of umbilical artery Doppler indices in the second half of pregnancy. Am J Obstet Gynecol. 2005; 192(3):937-44.

5. Acharya G, Wilsgaard T, Berntsen GK, Maltau JM, Kiserud T. Reference ranges for serial measurements of blood velocity and pulsatility index at the intra-abdominal portion, and fetal and placental ends of the umbilical artery. Ultrasound Obstet Gynecol. 2005;26(2): 162-9.

6. Kessler J, Rasmussen S, Hanson M, Kiserud T. Longitudinal reference ranges for ductus venosus flow velocities and waveform indices. Ultrasound Obstet Gynecol. 2006;28(7):890-8.

7. Turan S, Turan OM, Berg C, Moyano D, Bhide A, Bower S, et al. Computerized heart rate analysis, Doppler ultrasound and biophysical profile score in the prediction of acid-base status of growth-restricted fetuses. Ultrasound Obstet Gynecol. 2007;30(5):750-6.

8. Tarzamni MK, Nezami N, Sobhani N, Eshraghi N, Tarzamni M, Talebi Y. Nomograms of Iranian fetal middle cerebral artery Doppler waveforms and uniformity of their pattern with other populations' nomograms. BMC Pregnancy Childbirth. 2008;8:50.

9. Flo K, Wilsgaard T, Acharya G. Relation between utero-placental and feto-placental circulations: a longitudinal study. Acta Obstet Gynecol Scand. 2010;89(10):1270-5.
10. Kaponis A, Harada T, Makrydimas G, Kiyama T, Arata K, Adonakis $G$, et al. The importance of venous Doppler velocimetry for evaluation of intrauterine growth restriction. J Ultrasound Med. $2011 ; 30(4): 529-45$

11. Society for Maternal-Fetal Medicine Publications Committee, Berkley E, Chauhan SP, Abuhamad A. Doppler assessment of the fetus with intrauterine growth restriction. Am J Obstet Gynecol. 2012;206(4):300-8.

12. Kalache KD, Dückelmann AM. Doppler in obstetrics: beyond the umbilical artery. Clin Obstet Gynecol. 2012;55(1):288-95.

13. Tongprasert F, Srisupundit K, Luewan S, Wanapirak C, Tongsong T. Normal reference ranges of ductus venosus Doppler indices in the period from 14 to 40 weeks' gestation. Gynecol Obstet Invest. 2012;73(1):32-7.

14. Robinson HP, Fleming JE. A critical evaluation of sonar "crown-rump length" measurements. Br J Obstet Gynaecol. 1975;82(9):702-10.

15. Hadlock FP, Harrist RB, Carpenter RJ, Deter RL, Park SK. Sonographic estimation of fetal weight. The value of femur length in addition to head and abdomen measurements. Radiology. 1984;150(2):53540.

16. WFUMB Symposium on Safety of Ultrasound in Medicine. Conclusions and recommendations on thermal and non-thermal mechanisms for biological effects of ultrasound. Ultrasound Med Biol. 1998;24 Suppl 1:S1-58.

17. Royston P, Wright EM. How to construct 'normal ranges' for fetal variables. Ultrasound Obstet Gynecol. 1998;1 1(1):30-8.

18. Fieuws S, Verbeke G, Molenberghs $G$. Random-effects models for multivariate repeated measures. Stat Methods Med Res. 2007; 16(5):387-97

19. Young IT. Proof without prejudice: use of the Kormogorov-Smirnov test for the analysis of histograms from flow systems and others sources. J Histochem Cytochem. 1977;25(7):935-41. 